\title{
Collaboration space division in collaborative product development based on a genetic algorithm
}

\author{
Xueming Qian ${ }^{1} \cdot$ Yanqiao $\mathrm{Ma}^{1} \cdot$ Huan Feng ${ }^{1}$
}

Received: 16 August 2017 / Accepted: 11 January 2018/ Published online: 8 February 2018

(C) The Author(s) 2018. This article is an open access publication

\begin{abstract}
The advance in the global environment, rapidly changing markets, and information technology has created a new stage for design. In such an environment, one strategy for success is the Collaborative Product Development (CPD). Organizing people effectively is the goal of Collaborative Product Development, and it solves the problem with certain foreseeability. The development group activities are influenced not only by the methods and decisions available, but also by correlation among personnel. Grouping the personnel according to their correlation intensity is defined as collaboration space division (CSD). Upon establishment of a correlation matrix (CM) of personnel and an analysis of the collaboration space, the genetic algorithm (GA) and minimum description length (MDL) principle may be used as tools in optimizing collaboration space. The MDL principle is used in setting up an object function, and the GA is used as a methodology. The algorithm encodes spatial information as a chromosome in binary. After repetitious crossover, mutation, selection and multiplication, a robust chromosome is found, which can be decoded into an optimal collaboration space. This new method can calculate the members in sub-spaces and individual groupings within the staff. Furthermore, the intersection of sub-spaces and public persons belonging to all sub-spaces can be determined simultaneously.
\end{abstract}

Keywords MDL · Genetic algorithm · Collaboration space division · Chromosome

\section{Introduction}

The advance in the global environment, rapidly changing markets, and information technology has created a new stage for design. On the other side, the features of nowadays' rival environment compels corporations to seek innovative approaches to trade, such as the speed of product design, manufacture, and the requirements for lower operating costs (Babazadeh et al. 2012). Enterprises should be able to control distributed operations around the world to maintain their competitive advantages (Hong 2004). In this environment, one strategy that corporations want to succeed is Collaborative Product Development (CPD). There are some similar terms in the literature, such as collaborative engineering (Park and Cutkosky 1999),

Xueming Qian

bearqianxueming@163.com

1 School of Mechatronic Engineering, Xi'an Technological University, No. 2, Middle Xuefu Road,

Xi'an 710021, Shaanxi Province, China collaborative design ( $\mathrm{Li}$ et al. 2004), and collaborative product commerce (Morris 2002). Each term emphasizes different aspects and applications. They have made significant efforts in collaborative product development research over the past few decades. In the development of a collaborative information platform, the majority of them are very good at using information technology to increase collaboration (Li and Qiu 2006). However, collaborative product development involves many aspects, including stakeholders with various purposes and backgrounds. Hence, the development team's activities are influenced by many factors, including the usable tools, methods, strategy decisions and interpersonal relevance (Lu et al. 2000). In collaborative product development, correlation among personnel should also be considered.

So from this point of view, there are three important questions: firstly, how to develop the correlation among personnel. Secondly, how to group the personnel into different teams according to the correlation. And thirdly, how to determine which engineers will belong to the different teams. The virtual space for the whole collaborative 
personnel is defined as the collaboration space (CS), and the space for one team is named as a sub-space. In diverse tasks, based on the interdependent intensity, grouping the personnel is defined as collaboration space division. In this paper, we obtain the correlation among personnel by Correlation Matrix (CM) and divide the collaboration space to determine which of the engineers belong to the different teams by GA.

The rest of the paper is presented as follows. The next section provides a quick review of related literature. The correlation matrix and collaboration space are studied in Sect. 3. We then propose an objective function for CSD in Sect. 4, and introduce the CS division based on GA in Sect. 5. An example of CSD for impeller development is described in Sect. 6, and conclusions are described in Sect. 7.

\section{Literature review}

Implementing CPD requires the introduction of revolutionary changes in technology and organizations. In the past 10 years, a great number of collaborative environments were presented. These employees focused on building a virtual collaborative environment based on virtual reality (Summers et al. 1999; Singhal et al. 2004; Frecon and Noeu 1998). In the task, the most difficult problem is the lack of a common vocabulary for describing virtual collaborative environments. In the process of building collaborative design environments, the employees researched the ontology (Waterson and Preece 1999; Roche 2000; Navigli and Velardi 2003). In Ling Ling's research (2007), the collaborative design environment, where the design agents can deal with all kinds of problems in the multi-agent system by their specific area of knowledge. Then, in the CPD environment, there is an ontologybased knowledge integration framework. It can improve the ability to deal with the challenging issues.

Based on the result of the TEAM project, May et al. (2004) presented a case study. In the context of reality, they discussed the technical factors, human factors and base structure for concurrent engineering. The purpose of this article is to research the effect of advanced IT\&T (information technology and telecommunications), and it sustained the concurrent engineering during the product introduction.

The traditional design knowledge cannot play an important role in the intricate product development, because the intricacy of the product is growing. So, they need flexible design concepts. Chiang et al. (2006) put forward a comprehensive framework for designing knowledge representation. In the design inference processes, they develop an intelligent system based on knowledge, to promote dynamic design reasoning, adoption and collaboration of knowledge. In this research, the capacities of intelligent collaborative design reasoning have been enhanced. They provided KBIS with a conceptual architecture, and it enabled the collaborative design community to determine practicable design specifications and combine all of the sub-system's designs for the product.

However, a collaborative environment is not the whole of CPD. As the CPD's design chains differs from traditional product development, cooperative enterprises still manage their product development processes in a low efficiency manner during the collaboration processes between dislocated workers. To gain a competitive advantage, the company has begun to adopt the design chain, shortening the time between design of product and time to marketplace (Twigg 1995). Mentioned the conception of the design chain, Twigg (1995) researched the design information exchange with suppliers and customers, through which the design information can be compared between the inter-organizational project management engineering design. With the combination of different enterprise design documents, a mutual influence of the chain system has been formed. System members benefit by sharing the development costs, and the system named "design chain". It has a wide range of applications, especially at various stages of new product development, such as creativity, preliminary assessment, product engineering, product testing and release, and so forth (Cooper 1994). Some industries incorporate the design chain ideas into new product development, such as the automobile and motorcycle industry. In the design phase, the design chain involves the cooperation between diverse corporations. During the process of product development, the participants can also take part in the discussions with other designers and manufacturers. They call it collaborative design process (Wang et al. 2002). Wognum et al. (2002) thought highly of cooperation between clients and suppliers in product development. The advantages of collaborative design include reducing the costs of new product conception, reducing research time, and enhancing competitive power (Huang et al. 2003). The collaborative design process needs to determine customer requirements and product specifications. According to these requirements and specifications, product developers determine the basic tasks in development. In the distributed environment, team members are a CPD each component. The whole design project must be interwoven between groups, even in the design process. When completing their activities, members use different rules to maintain product consistency.

To enhance the information transfer between organizations, a corporation should reexamine and redesign their 
process. Barrett and Konsynski (1982) presented the main motivation for inter-organizational messaging and sharing is to reduce costs of transaction. Lee and Chen (1996) argued that designers and manufacturers need to communicate and share information effectively throughout the process of collaborative design project. Corbett et al. (1999) emphasizes that the efficiency of supply chain management can be enhanced through the information sharing and coordination decisions among supply chain partners. Kim et al. (2006) developed a framework, which can be used for product information sharing in enterprises collaborative design project. To share the information, Choi et al. (2005) proposed constructing a product design chain collaboration framework.

Thus, information sharing seems like a design chain "lubricant", and it can promote inter-organizational collaboration. A corporation should boost operational processes to facilitate collaboration among personnel, when they deal with organizational behaviour. They can work together to develop the products.

Then, there are some important questions: who should share the same information and who should be grouped into the same team? In this paper, following the CPD principle, the correlation among personnel is derived from employee's tasks and activities in the collaborative product development. According to the correlation, employees are divided into sub-collaborative spaces. Employees in the same sub-spaces share the same information while redesigning and reexamining. The next section describes the method for obtaining correlation among personnel in detail.

\section{Correlation matrix and collaboration space}

\section{Correlation matrix}

Consider a CPD process that has $\mathrm{n}$ sub-tasks, and $\mathrm{m}$ engineers attending to it. The cooperation times of the subtasks are denoted as $\left\{N_{1}, N_{2}, \cdots, N_{n}\right\}$. Firstly, we establish the interdependent matrix for engineers and sub-tasks, in which the row shows the sub-tasks and the column shows the engineers. The element $V_{i j}$ of the matrix indicates that the engineer operated collaboratively $V_{i j}$ times in the subtask. Table 1 shows the personnel-task interdependent matrix that is made up of 12 engineers and 18 sub-tasks.

In the matrix, 12 engineers, who participate in the impeller development process in seven different roles for solving 18 sub-tasks, complete their operations associated to the $i$ th sub-task $V_{i j}$ times. Each column vector indicates the focus of one engineer. For example, client Liu's column vector $\left[\begin{array}{llllllllllll}7 & 6 & 6 & 0 & 6 & 8 & 11 & 0 & 2 & 0 & 2 & 0\end{array}\right.$ $\left.\begin{array}{llllll}0 & 0 & 0 & 2 & 0 & 0\end{array}\right]^{T}$ is different from process engineer Zhu's vector $\left[\begin{array}{llllllllllll}0 & 2 & 0 & 0 & 0 & 0 & 0 & 0 & 5 & 7 & 2 & 8\end{array}\right.$ $\left.\begin{array}{llllll}5 & 3 & 4 & 0 & 5 & 9\end{array}\right]^{T}$. The difference indicates that, client Liu is focused on the design performance while process engineer Zhu pays much more attention to machinability and testing performance. Considering the collaborative operation times, we normalize interdependent matrix columns in the personnel-task matrix $A$ as shown in Table 2 . In Table 2 the column vector represents the participation of a given engineer in each task (i.e., $A_{i j}$ shows the participation of the engineer $j$ in the task $i$ ). Two very similar column vectors is an indication of strong correlation between the corresponding two engineers. The correlation coefficient between two engineers is defined as:

$S_{i j}=1-\sqrt{\sum_{k=1}^{n}\left(A_{i k}-A_{j k}\right)^{2}}$.

After calculating the pairwise correlation coefficients for all pairs of engineers, the correlation matrix $(\mathrm{CM})$ for engineers is constructed as follows: $S=[S]_{i j}$ (as shown in Table 3):

$\mathrm{CM}$ is a symmetric matrix, and the diagonal elements are all valued $1\left(S_{i i}=1\right)$. To simplify the relation among personnel, the $\mathrm{CM}$ is transformed into a zero-one matrix by a gate. Suppose the gate value is 0.5 , then the matrix in Table 3 is transformed into that of Table 4. The matrix $S$ implies the collaboration space (CS). Analysis of the CS is stated in what follows.

\section{Collaboration space analysis}

According to the correlative intensity of the engineers, the CS has many division projects. The first problem will be to choose an optimization standard to establish the optimal CS. The standard chosen in this paper is as follows:

The Algorithm should be able to give an optimal division of the CS.

The Algorithm should be able to ascertain the public space.

The Algorithm should be able to determine the public engineers that belong to a given sub-space.

\section{Collaboration space dimensions and topological structure}

The correlation matrix gives the correlation between collaborative members in different fields. The correlative complexity increases with a growth in collaboration members. We first establish a $12 \times 12$ correlation matrix, which is depicted in Table 4. Second, analysis of the row and column transformations suggests that the CS has a three-dimensional tetrahedron structural, as shown in 


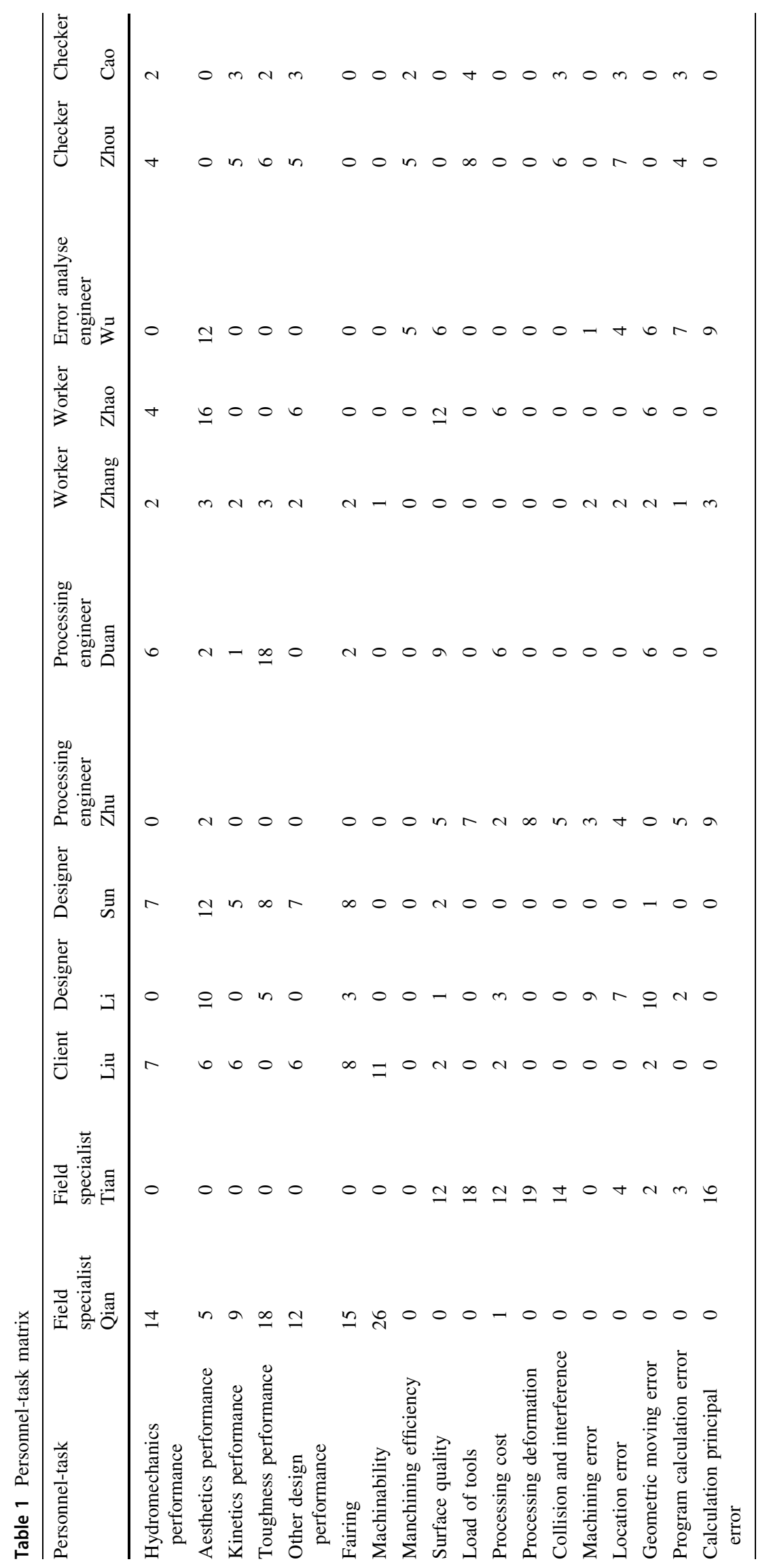




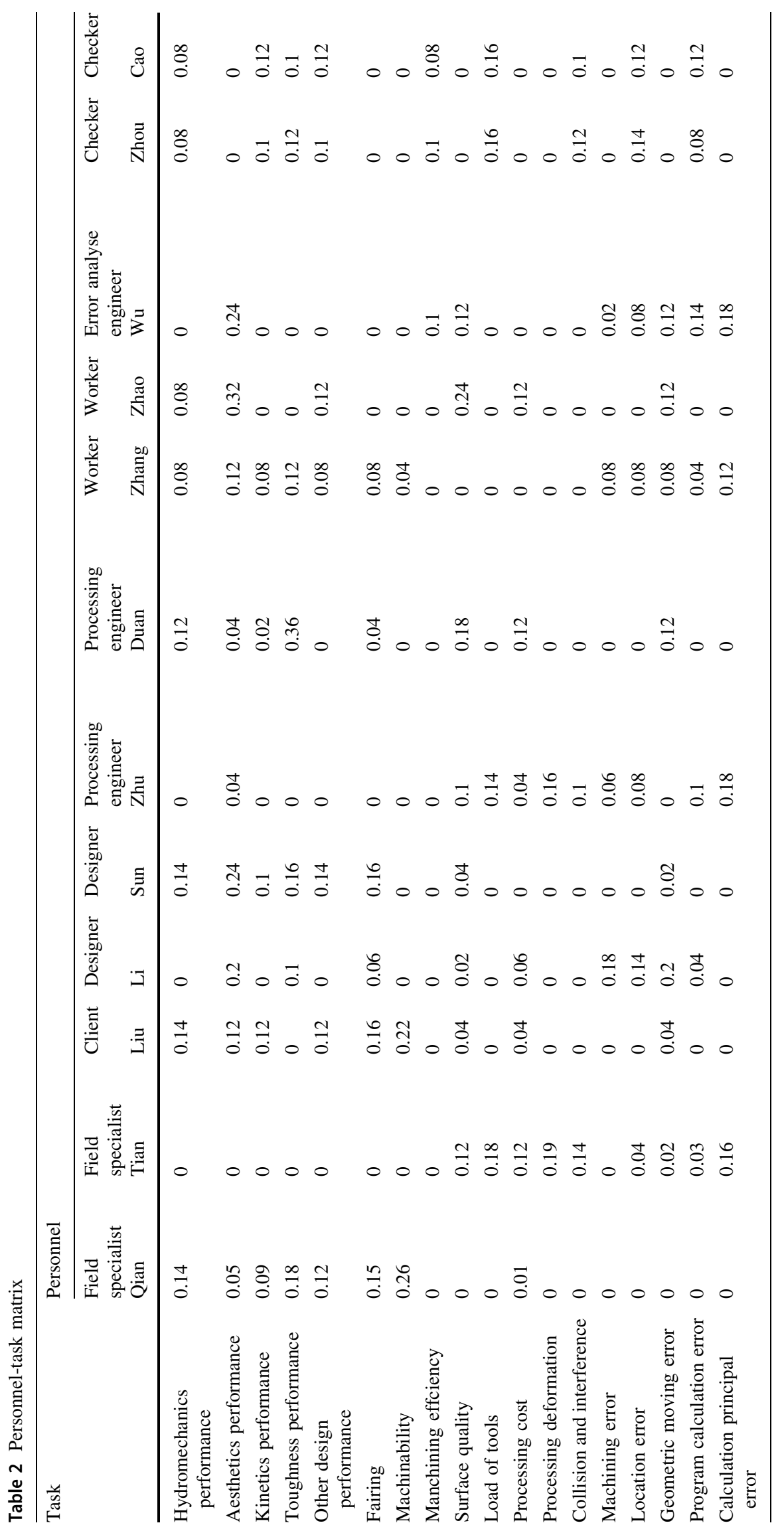


Table 3 Correlation matrix, describing correlations among personnel

\begin{tabular}{llllllllllllll}
\hline & & $\mathbf{1}$ & $\mathbf{2}$ & $\mathbf{3}$ & $\mathbf{4}$ & $\mathbf{5}$ & $\mathbf{6}$ & $\mathbf{7}$ & $\mathbf{8}$ & $\mathbf{9}$ & $\mathbf{1 0}$ & $\mathbf{1 1}$ & $\mathbf{1 2}$ \\
1. Qian & $\mathbf{1}$ & 1 & 0.4 & 0.8 & 0.4 & 0.8 & 0.5 & 0.4 & 0.8 & 0.5 & 0.5 & 0.7 & 0.7 \\
2. Tian & $\mathbf{2}$ & 0.4 & 1 & 0.5 & 0.5 & 0.5 & 0.9 & 0.5 & 0.6 & 0.5 & 0.6 & 0.6 & 0.6 \\
3. Liu & $\mathbf{3}$ & 0.8 & 0.5 & 1 & 0.6 & 0.7 & 0.5 & 0.5 & 0.7 & 0.6 & 0.3 & 0.2 & 0.4 \\
4. Li & $\mathbf{4}$ & 0.4 & 0.5 & 0.6 & 1 & 0.6 & 0.5 & 0.7 & 0.5 & 0.6 & 0.7 & 0.4 & 0.4 \\
5. Sun & $\mathbf{5}$ & 0.8 & 0.5 & 0.7 & 0.6 & 1 & 0.4 & 0.5 & 0.7 & 0.6 & 0.5 & 0.6 & 0.6 \\
6. Zhu & $\mathbf{6}$ & 0.5 & 0.9 & 0.5 & 0.5 & 0.4 & 1 & 0.5 & 0.6 & 0.5 & 0.7 & 0.3 & 0.3 \\
7. Duan & $\mathbf{7}$ & 0.4 & 0.5 & 0.5 & 0.7 & 0.5 & 0.5 & 1 & 0.4 & 0.5 & 0.6 & 0.5 & 0.5 \\
8. Zhang & $\mathbf{8}$ & 0.8 & 0.6 & 0.7 & 0.5 & 0.7 & 0.6 & 0.4 & 1 & 0.5 & 0.7 & 0.7 & 0.7 \\
9. Zhao & $\mathbf{9}$ & 0.5 & 0.5 & 0.6 & 0.6 & 0.6 & 0.5 & 0.7 & 0.6 & 1 & 0.7 & 0.5 & 0.5 \\
10. Wu & $\mathbf{1 0}$ & 0.5 & 0.6 & 0.3 & 0.7 & 0.5 & 0.7 & 0.6 & 0.7 & 0.7 & 1 & 0.5 & 0.4 \\
11. Zhou & $\mathbf{1 1}$ & 0.7 & 0.6 & 0.3 & 0.4 & 0.6 & 0.3 & 0.5 & 0.7 & 0.5 & 0.5 & 1 & 0.1 \\
12. Cao & $\mathbf{1 2}$ & 0.7 & 0.6 & 0.4 & 0.4 & 0.6 & 0.3 & 0.5 & 0.7 & 0.5 & 0.4 & 0.1 & 1
\end{tabular}

Table 4 Correlation matrix, describing correlations among personnel

\begin{tabular}{rrrrrrrrrrrrr}
\hline & $\mathbf{1}$ & $\mathbf{2}$ & $\mathbf{3}$ & $\mathbf{4}$ & $\mathbf{5}$ & $\mathbf{6}$ & $\mathbf{7}$ & $\mathbf{8}$ & $\mathbf{9}$ & $\mathbf{1 0}$ & $\mathbf{1 1}$ & $\mathbf{1 2}$ \\
1 & 1 & 0 & 1 & 0 & 1 & 0 & 0 & 1 & 0 & 0 & 1 & 1 \\
$\mathbf{2}$ & 0 & 1 & 0 & 0 & 0 & 1 & 0 & 1 & 0 & 1 & 1 & 1 \\
$\mathbf{3}$ & 1 & 0 & 1 & 1 & 1 & 0 & 0 & 1 & 1 & 0 & 0 & 0 \\
$\mathbf{4}$ & 0 & 0 & 1 & 1 & 1 & 0 & 1 & 0 & 1 & 1 & 0 & 0 \\
$\mathbf{5}$ & 1 & 0 & 1 & 1 & 1 & 0 & 0 & 1 & 1 & 0 & 1 & 1 \\
$\mathbf{6}$ & 0 & 1 & 0 & 0 & 0 & 1 & 0 & 1 & 0 & 1 & 0 & 0 \\
$\mathbf{7}$ & 0 & 0 & 0 & 1 & 0 & 0 & 1 & 0 & 1 & 1 & 1 & 1 \\
$\mathbf{8}$ & 1 & 1 & 1 & 0 & 1 & 1 & 0 & 1 & 0 & 1 & 1 & 1 \\
$\mathbf{9}$ & 0 & 0 & 1 & 1 & 1 & 0 & 1 & 0 & 1 & 1 & 0 & 0 \\
$\mathbf{1 0}$ & 0 & 1 & 0 & 1 & 0 & 1 & 1 & 1 & 1 & 1 & 0 & 0 \\
$\mathbf{1 1}$ & 1 & 1 & 0 & 0 & 1 & 0 & 1 & 1 & 0 & 0 & 1 & 1 \\
$\mathbf{1 2}$ & 1 & 1 & 0 & 0 & 1 & 0 & 1 & 1 & 0 & 0 & 1 & 1 \\
\hline
\end{tabular}

Fig. 1a. The blue field in the figure depicts the four collaboration sub-spaces $\{\mathrm{AA}, \mathrm{BB}, \mathrm{CC}, \mathrm{DD}\}$. Each sub-space has strong internal relationships and weak external relationships. The grey fields depict the cross relationships between $\{\mathrm{AA}, \mathrm{BB}, \mathrm{CC}, \mathrm{DD}\}$. The topological structure of the sub-space is depicted in Fig. 1b. If the engineers and the interdependent intensity are the same in each subspace, the sub-space AA will expand to envelope the whole $\mathrm{CS}$. If the parts of the personnel of the sub-spaces are same, the whole CS will be divided into several sub-spaces.

Suppose we have an original CS matrix, $S=\left[S_{i j}\right]$. The original space can be acquired by transforming the optimal matrix. The original CS, $S_{n \times n}\left(S_{i j} \in\{0,1\}\right)$, can be depicted as: $S_{n \times n}=S_{n \times n}+M_{n \times n}$

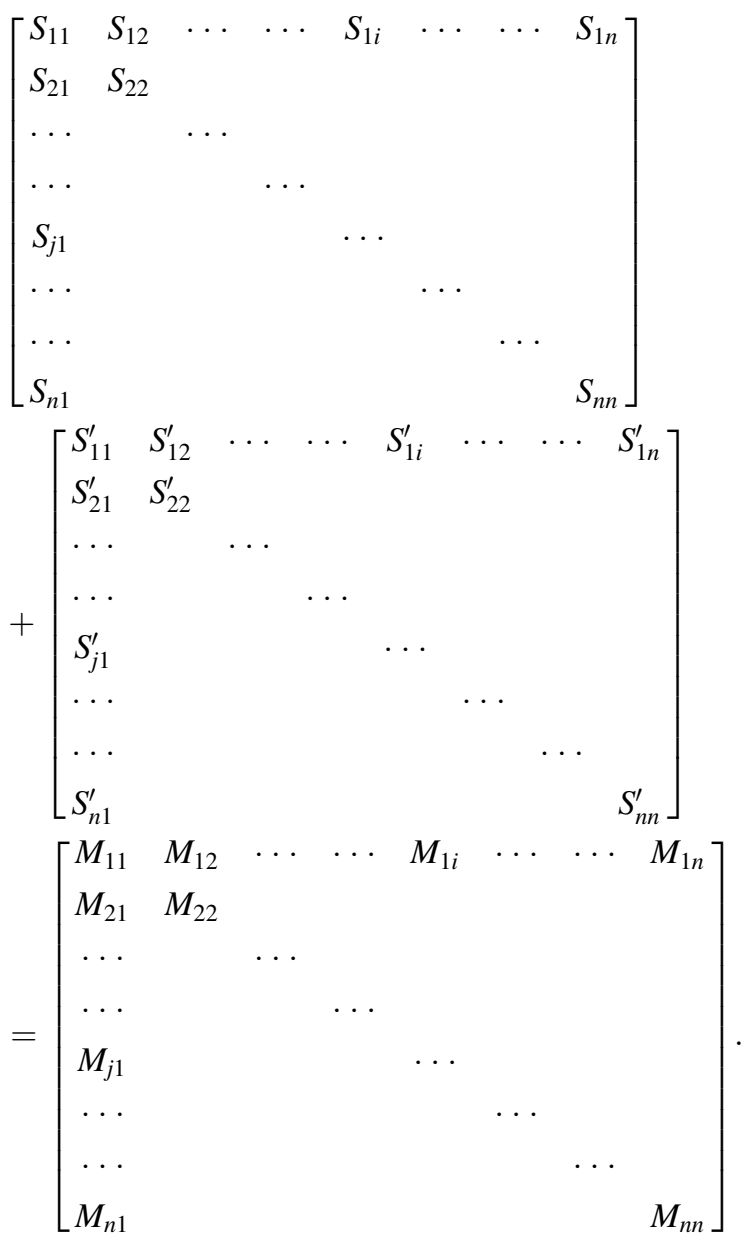

Here, $S_{n \times n}^{\prime}$ denotes the optimal space description matrix, $S_{i j}^{\prime} \in\{0,1\} . M_{n \times n}$, the named space transformation matrix, describes the differences between the description of two matrixes, $M_{i j}=S_{i j}-S_{i j}^{\prime}, M_{i j} \in\{-1,0,1\}$. When $M_{i j}=1$, can be interpreted as implying a transformation for the ijth element of the matrix $S_{n \times n}$, of the type: $S_{i j}=1 \rightarrow S_{i j}^{\prime}=0$. When $M_{i j}=-1$, the implied transformation is of type 


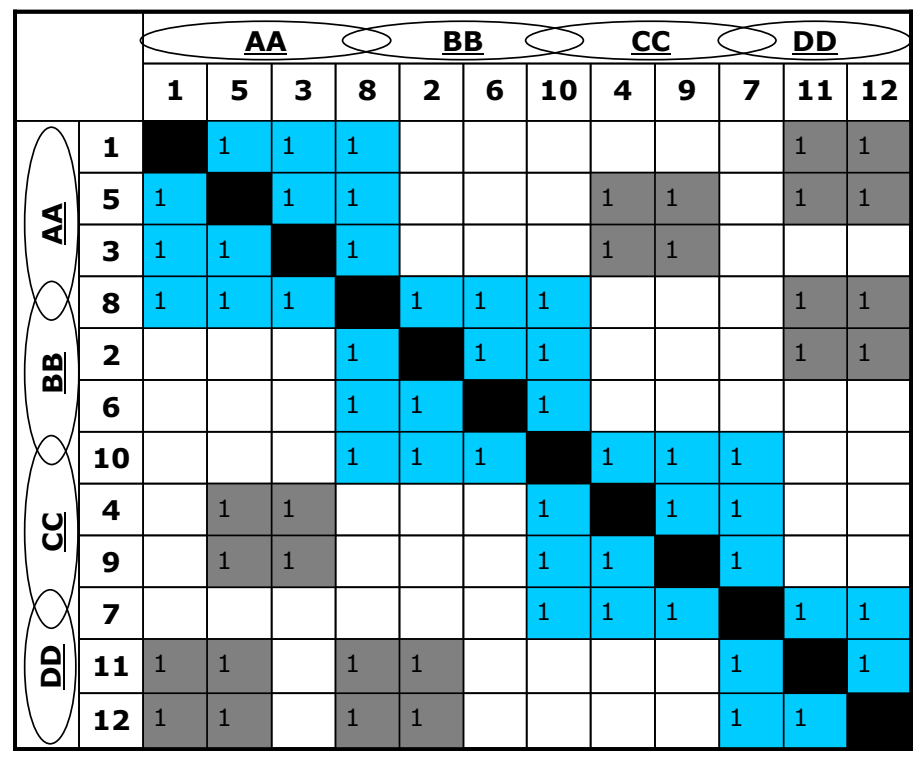

(a)

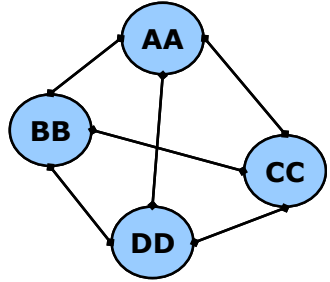

(b)

Fig. 1 Collaborative space and its corresponding topological structure

$S_{i j}=0 \rightarrow S_{i j}^{\prime}=1$. When $M_{i j}=0$, there is no transformation implied for the $i j$ th element.

\section{MDL principle and the optimal objective function of collaboration space division}

\section{Introduction of the MDL principle}

There is a model which describes a CS $S=\left[S_{i j}\right]$. The "model" means a prescription of which collaboration space the engineer belongs to. According to section "Collaboration space analysis", the description of the original CS consists of two sections: the description of optimal space and the description of space transformations. In its full demarcation, A CS enlarges the length of the optimal space description, while the description length of the space transformation will be reduced. In contrast, if the length of optimal space description is reduced, the description length of the space transformation will be enlarged. According to the following sender-receiver example, the plan will be more easily understood.

Suppose the sender has the given data set that the receiver requires. There is a model that can approximate a given set of data. And the sender sends the model to the recipient for the first time. The sender is also required to send the data in order that recipients get exactly the same data set. And the data are misdescribed by the model sent earlier. The model description will be very short if it is too brief. In fact, there are many data mismatches that make the description longer. At the same time, mismatched data will be reduced in complex models, but the description of model will be longer.

However, the above dilemma can be dealt with the MDL (minimum description length) principle (Lutz 2002). MDL suggests that, in all of the depicted schemes, we choose to use the minimal length to describe both the optimal space information and the space transformations. There are two key points worth noting. When the CS is divided, the space encoding information should have unique decodability, and the length of the code should incarnate the space complexity.

\section{The objective function for CS optimization}

\section{Code and description length of optimal mode}

All of the collaboration engineers are viewed as nodes for the binary encoding. The encoding is made up of three parts: the space serial number, the node number and the node clustering. Each collaborative sub-space starts with the assigned space number. The second part, the node number, describes the amount of the nodes. The third part describes the nodes in the sub-space. In Fig. 2, there is a description of a sub-space model. The first part, 111, refers to sub-space number 8 . The second part, 11111, indicates that there are 32 nodes in the space, and the third part, $\{01001,01010,01111\}$, means that $\{01001,01010,01111\}$ belongs to the No. 8 sub-space.

The whole collaboration space is a set of sub-spaces. The CS description length can be calculated as follows. Suppose we have a model in which there is $n_{c}$ sub-spaces 
Fig. 2 Description of sub-space 1

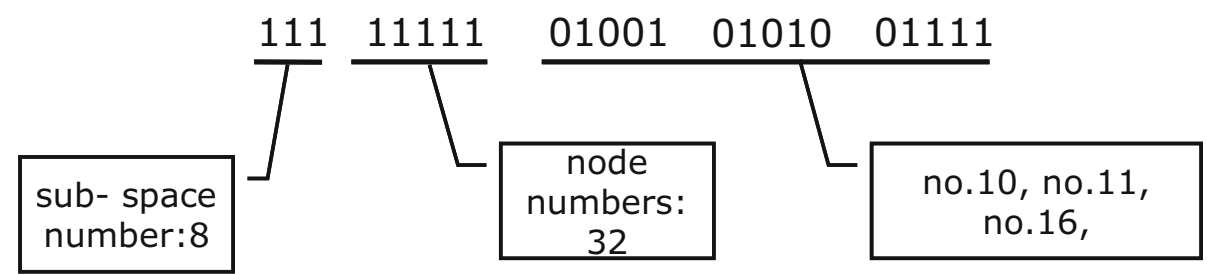

and $n_{n}$ nodes. The numbers of nodes in the sub-spaces are $\left\{c l_{i} \mid i=1 \cdots \in n_{c}\right\}$. Then the $i$ th sub-space's length is given by $\log n_{c}+\log n_{n}+c l_{i} \times \log n_{n}$, and the whole CS's length is as follows:

$\sum_{i=1}^{n_{c}}\left(\log n_{c}+\log n_{n}+c l_{i} \times \log n_{n}\right)$,

the logarithm base is 2 .

\section{Code and description length of the space transform}

The space transform is carried out by the $M$ matrix (as described in 1.2). When $S_{i j} \neq S_{i j}^{\prime}, M_{i j} \neq 0$, a description is needed to indicate $M_{i j}$. The description length about $M_{i j} \neq 0$ and the site is given by $\log n_{n}+\log n_{n}+1$, among which it takes $\log n_{n}+\log n_{n}$ bits to indicate $i$ and $j$, and 1 bit to indicate whether $M_{i j}=1$ or $M_{i j}=-1$.

Let's define a transform model $M . M$ includes two parts, $m_{1} \quad$ and $\quad m_{2}$, with $m_{1}=\left\{(i, j) / M_{i j}=1\right\}, m_{2}=$ $\left\{(i, j) / M_{i j}=-1\right\}$. Then the description length for the space transform model $M$ is as follows:

$\sum_{(i, j) \in m_{1}}\left(\log n_{n}+\log n_{n}+1\right)+\sum_{(i, j) \in m_{2}}\left(\log n_{n}+\log n_{n}+1\right)$.

\section{The objective function}

According to the MDL principle, the optimal CSD scheme is the one which uses the minimal length for describing the optimal space information and the model of space transform information. The object function is depicted as follows:

$$
\begin{aligned}
f\left(M, S^{\prime}\right)= & {\left[n_{c} \log n_{c}+n_{c} \log n_{n}+\log n_{n} \sum_{i=1}^{n_{c}} c l_{i}\right] } \\
& +\sum_{(i, j) \in m_{1}}\left(\log n_{n}+\log n_{n}+1\right) \\
& +\sum_{(i, j) \in m_{2}}\left(\log n_{n}+\log n_{n}+1\right) .
\end{aligned}
$$

\section{The CS division based on a GA}

\section{The characteristics of GA}

The Genetic algorithm (GA) is a global optimization search algorithm, which based on natural heredity and optimal selection. The GA is a particularly advantageous algorithm for their simplicity and robustness. They are suitable for concurrent processing as well as a wide range of application. However, it is only a general search method. For every problem, there is no universal GA capable of solving problems superiorly. Meanwhile, the specific problems in chromosome encoding and genetic operation are always needed (Dao et al. 2017) . The main characteristic of the GA is that they can search for the optimal solution by point-cluster, rather than point-by-point. The GA also only use fitness functions for the problem without any other precondition or assistant information. They use a random conversion rule rather than an assured rule. In this paper, The GA is used in collaborative space division.

\section{Composition and decoding of chromosomes}

As indicated in 1.2, there are three space-dividing criteria used in searching the optimal scheme of CSD, judging the common space and detecting the engineers belonging to the public. In addition, the topological structure of the CS described by the chromosome must be unique.

In this paper, binary encoding is adopted, and the length of the chromosome is $c \cdot n_{n}$ bits. Here, $c$ is defined as maximal number of sub-spaces, and $n_{n}$ is the number of nodes (engineers). $x+y \cdot n_{n}$ bits form a piece of the gene which indicates that the node $\mathrm{x}$ belongs to the sub-space $\mathrm{y}$. Suppose the maximal number of sub-spaces is $c=5$, the tetrahedral topological structure of the CS chromosome (described in Fig. 3) is as follows:

The chromosome in Fig. 3 can be decoded as in Table 5:

\section{Algorithm organizations}

In this paper, the GA is adopted with fixed crossover, mutation probability, and $\lambda+\mu$ selection. In what follows, the population of the chromosome is $n=\lambda$, the crossover probability is $P_{c}=\mu$, the mutation probability is $P_{m}=\alpha$, 


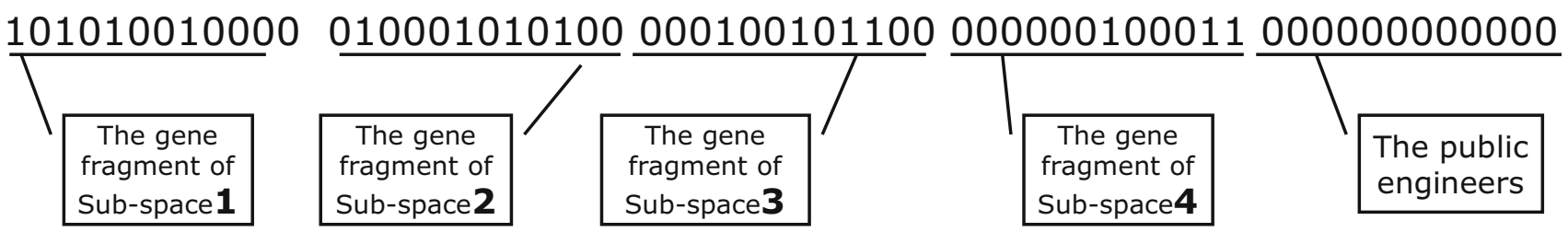

Fig. 3 Composition of chromosome

Table 5 Decoding of the chromosome

\begin{tabular}{lllllllllllll}
\hline Node & $\mathbf{1}$ & $\mathbf{2}$ & $\mathbf{3}$ & $\mathbf{4}$ & $\mathbf{5}$ & $\mathbf{6}$ & $\mathbf{7}$ & $\mathbf{8}$ & $\mathbf{9}$ & $\mathbf{1 0}$ & $\mathbf{1 1}$ & $\mathbf{1 2}$ \\
Sub-space: 1 & 1 & 0 & 1 & 0 & 1 & 0 & 0 & 1 & 0 & 0 & 0 & 0 \\
Sub-space: 2 & 0 & 1 & 0 & 0 & 0 & 1 & 0 & 1 & 0 & 1 & 0 & 0 \\
Sub-space: 3 & 0 & 0 & 0 & 1 & 0 & 0 & 1 & 0 & 1 & 1 & 0 & 0 \\
Sub-space: 4 & 0 & 0 & 0 & 0 & 0 & 0 & 1 & 0 & 0 & 0 & 1 & 1 \\
$\begin{array}{l}\text { The public } \\
\text { engineers }\end{array}$ & 0 & 0 & 0 & 0 & 0 & 0 & 0 & 0 & 0 & 0 & 0 & 0 \\
\hline
\end{tabular}

the maximal number of clusters is $t=\beta$, the maximal number of sub-spaces is equal to $n_{c}$. The process of searching for the optimal result using the above parameters is as follows:

\section{Choosing the initial population}

Each chromosome is made up of a collection of $n_{c}$ gene segments, each of whose length is $n_{c}$. Each chromosome indicates a specific CSD program. The $\lambda$ chromosomes are then constructed at random, the initial population is:

$A_{0}^{(t)}=\left\{\left(M_{0 i}^{(t)}, S_{0 i}^{(t)}\right) \mid i=1,2, \cdots, \lambda\right\}$,

\section{Crossover operation}

In predefining the maximal number of sub-spaces as $n_{c}$, and the number of nodes as $n_{n}$, the chromosome will be a binary string of $n_{c} \cdot n_{n}$ bits. The process of crossover operation includes the following steps: choosing two chromosomes individually with the same probability $p_{c}$ from $A 1(t)$, choosing a random position in the $n_{c} \cdot n_{n}$ bits, and exchanging the codes of two chromosomes in crossover position. The example is as in Fig. 4:

The process is repeated, until $\mu$ offspring chromosomes are produced.

$A 1(t)=\left\{\left(M_{1 i}^{(t)}, S_{1 i}^{(t)}\right) \mid i=1,2, \cdots, \mu\right\}$.

\section{Mutation operation}

The mutation occurs according to mutation probability $p_{m}$. First, choose one bit in a chromosome from population $A 1(t)$ randomly (a corresponding binary value) according to mutation probability $p_{m}$. Secondly, invert the value of this bit (from 0 to 1 , or from 1 to 0 ). After the crossover and mutation operations, the transitional population is formed:

$S_{0 i}^{\prime(t)}=\left\{\left.\left(S_{1 i}^{\prime} S_{2 i}^{\prime} \cdots S_{m i}^{\prime} \cdots S_{n_{n^{i}}}^{\prime}\right)_{1}^{t}\left(S_{1 i}^{\prime} S_{2 i}^{\prime} \cdots S_{m i}^{\prime} \cdots S_{n_{n^{i}}}^{\prime}\right)_{2}^{t} \cdots\left(S_{1 i}^{\prime} S_{2 i}^{\prime} \cdots S_{m i}^{\prime} \cdots S_{n_{n^{i}}}^{\prime}\right)_{k}^{t} \cdots\left(S_{1 i}^{\prime} S_{2 i}^{\prime} \cdots S_{m i}^{\prime} \cdots S_{n_{n^{i}}}^{\prime}\right)_{n_{c}}^{t}\right|_{i=1} \cdots, \lambda\right\}$

$M_{0 i}^{(t)}=S-S_{0 i}^{(t)}$

$$
A 2(t)=\left\{\left(M_{2 i}^{(t)}, S_{2 i}^{\prime(t)}\right) \mid i=1,2, \cdots, \mu\right\}
$$

Fig. 4 Crossover operation 


\section{Calculation of the adaptability of individual chromosomes}

After crossover and mutation, a population with $\lambda+\mu$ chromosomes ( $\lambda$ parent chromosomes and $\mu$ offspring chromosomes) is formed. We then calculate the values of the $\lambda+\mu$ individual chromosome's description function (Sect. 4.2.3):

$$
\begin{aligned}
f\left(M_{i}^{(t)}, S_{i}^{(t)}\right)= & {\left[n_{c} \log n_{c}+n_{c} \log n_{n}+\log n_{n} \sum_{i=1}^{n_{c}} c l_{i}\right] } \\
& +\sum_{(i, j) \in m_{1}}\left(\log n_{n}+\log n_{n}+1\right) \\
& +\sum_{(i, j) \in m_{21}}\left(\log n_{n}+\log n_{n}+1\right) .
\end{aligned}
$$

Since the change of the objective function's value and the change of adaptability are opposite, we set up the function of adaptability as follows:

$G\left(M_{i}^{(t)}, S_{i}^{(t)}\right)=f_{\max }-f\left(M_{i}^{(t)}, S_{i}^{(t)}\right)$.

\section{Multiplication operation}

The chromosomes in the population will be ranked from largest to smallest according to the adaptability. Then, choosing $\lambda$ chromosomes according to adaptability from the chromosome pool $(\lambda+\mu$ chromosomes), and determining the proportion in the chromosome pool after multiplication with the following formula:

$p_{i}^{t}=G\left(M_{i}^{(t)}, S_{i}^{(t)}\right) / \sum_{i=1}^{\lambda} G\left(M_{i}^{(t)}, S_{i}^{(t)}\right)$,

the number of individuals with chromosome $\left(M_{i}^{(t)}, S_{i}^{\prime(t)}\right)$ in the chromosome pool after multiplication is:

$\lambda_{i}^{t}=p_{i}^{t} \lambda$.

The new $\lambda$ chromosomes are as follows in the chromosome pool after multiplication (where $\lambda$ is the number of the new individual chromosomes):

$A_{3}^{(t)}=\left\{\left(M_{3 i}^{(t)}, S_{3 i}^{(t)}\right) \mid i=1,2, \cdots, \lambda\right\}$,

$A_{0}^{(t+1)}=A_{3}^{(t)}$.

A new population is then formed. We repeat 5.3.2-5.3.5, until it is satisfies the maximal number of generations.

At this point, $S_{31}^{(t)}$ is the optimal scheme for collaboration space division.

\section{Case study}

The impeller is a complex product with free surface parts. In the development of an impeller, tasks like designing, processing and testing are carried out by designers, manufacturing engineers and testing engineers. Judging the correlations among various personnel and grouping them into collaborative sub-spaces is important to the development of a CSD program. In this section, the collaborative space division for impeller development is used to illustrate the method proposed above.

\section{Correlation matrix}

In this case study, the task of developing an impeller is divided into eighteen sub-tasks, which are aimed at performing eighteen different objectives. These objectives are hydromechanical performance, aesthetic performance, kinetic performance, toughness, other performance, fairing, machinability, machining efficiency, surface quality, load of tools, processing cost, processing deformation, collision and interference, machining error, geometric moving error, program calculation error and calculation principal error. The engineers can collaborate synchronously or asynchronously in performing these eighteen objectives. The activities of engineers in different objectives are recorded and shown in Fig. 5. Specialist Qian attended 14 times to the objective of hydromechanical performance, five times to aesthetic performance, nine times to kinetic performance, 18 times to toughness performance, 12 times to other performance, 15 times to fairing, 26 times to machinability and 1 time to the cost objective. The activity vector is thus $\left[\begin{array}{llllllllll}14 & 5 & 9 & 18 & 12 & 15 & 26 & 0 & 0 & 0\end{array}\right.$ $\left.\begin{array}{llllllll}1 & 0 & 0 & 0 & 0 & 0 & 0 & 0\end{array}\right]^{T}$. The activity vectors of other personnel are calculated in the same way to obtain a full personnel-task matrix. This matrix reflects the attentions of different engineers.

To compare the activity vectors, the columns of the matrix are normalized as shown in Fig. 6. After calculating the correlation coefficient using the formulae introduce in Sect. 3,

$S_{i j}=1-\sqrt{\sum_{k=1}^{n}\left(A_{i k}-A_{j k}\right)^{2}}$

The correlation matrix can be obtained (shown in Fig. 7). From the correlation matrix, the correlation intensity among personnel can be judged. As shown in Fig. 7, in this case study, the correlation coefficient between Qian and Tian is 0.44, while the one between Qian and Liu is 0.78. Thus, the relation between Qian and Tian is weaker than the one between Qian and Liu. 


\begin{tabular}{|c|c|c|c|c|c|c|c|c|c|c|c|c|}
\hline persounel-task matrix & $\mid \begin{array}{c}\text { Speciali ist } \\
\mathbf{q} \text { i an }\end{array}$ & $\underbrace{\text { specialist }}_{\text {tian }}$ & $\underset{\substack{\text { client } \\
\text { liu }}}{c}$ & $\begin{array}{c}\text { desi gner } \\
1 i\end{array}$ & $\begin{array}{c}\text { desi ener } \\
\text { sun }\end{array}$ & \begin{tabular}{|c|}
$\begin{array}{c}\text { process } \\
\text { engineer } \\
\text { zhu }\end{array}$ \\
\end{tabular} & \begin{tabular}{|c|} 
process \\
engineer \\
duan
\end{tabular} & $\begin{array}{c}\text { rorker } \\
\text { zhamg }\end{array}$ & $\begin{array}{c}\text { rorker } \\
\text { zhao }\end{array}$ & \begin{tabular}{|c|c|}
$\begin{array}{c}\text { errox } \\
\text { analysis } \\
\text { engineer } \\
\text { enu }\end{array}$ \\
\end{tabular} & $\begin{array}{c}\text { testing } \\
\text { engineer } \\
\text { zhouz }\end{array}$ & $\begin{array}{c}\text { testing } \\
\text { engineer } \\
\text { cao }\end{array}$ \\
\hline hyfomechartios & 14 & 0 & 7 & 0 & 7 & 0 & 6 & 2 & 4 & 0 & 4 & 2 \\
\hline esthetics & 5 & 0 & 6 & 10 & 12 & 2 & 2 & 3 & 16 & 12 & 0 & 0 \\
\hline kinetics & 9 & 0 & 6 & 0 & 5 & 0 & 1 & 2 & 0 & 0 & 5 & 3 \\
\hline toughness & 18 & 0 & 0 & 5 & 8 & 0 & 18 & 3 & 0 & 0 & 6 & 2 \\
\hline others & 12 & 0 & 6 & 0 & 7 & 0 & 0 & 2 & 6 & 0 & 5 & 3 \\
\hline fairing & 15 & 0 & 8 & 3 & 8 & 0 & 2 & 2 & 0 & 0 & 0 & 0 \\
\hline machinability & 26 & 0 & 11 & 0 & 0 & 0 & 0 & 1 & 0 & 0 & 0 & 0 \\
\hline efficiency & 0 & 0 & 0 & 0 & 0 & 0 & 0 & 0 & 0 & 5 & 5 & 2 \\
\hline surface quality & 0 & 12 & 2 & 1 & 2 & 5 & 9 & 0 & 12 & 6 & 0 & 0 \\
\hline load of tools & 0 & 18 & 0 & $\overline{0}$ & 0 & 7 & 0 & 0 & 0 & 0 & 8 & 4 \\
\hline processing cost & 1 & 12 & 2 & 3 & 0 & 2 & 6 & 0 & 6 & 0 & 0 & 0 \\
\hline deformation & 0 & 19 & 0 & 0 & 0 & 8 & 0 & 0 & 0 & 0 & 0 & 0 \\
\hline interference & 0 & 14 & 0 & 0 & 0 & 5 & 0 & 0 & 0 & 0 & 6 & 3 \\
\hline fachining error & 0 & 0 & 0 & 9 & 0 & 3 & 0 & 2 & 0 & 1 & 0 & 0 \\
\hline focation error & 0 & 4 & 0 & 7 & 0 & 4 & 0 & 2 & 0 & 4 & 7 & 3 \\
\hline Efometric error & 0 & 2 & 2 & 10 & 1 & 0 & 6 & 2 & 6 & 6 & 0 & 0 \\
\hline pregram err fr & 0 & 3 & 0 & 2 & 0 & 5 & 0 & 1 & 0 & 7 & 4 & 3 \\
\hline printipal frror & 0 & 16 & 0 & 0 & 0 & 9 & 0 & 3 & 0 & 9 & 0 & \\
\hline
\end{tabular}

Fig. 5 Activities of personnel

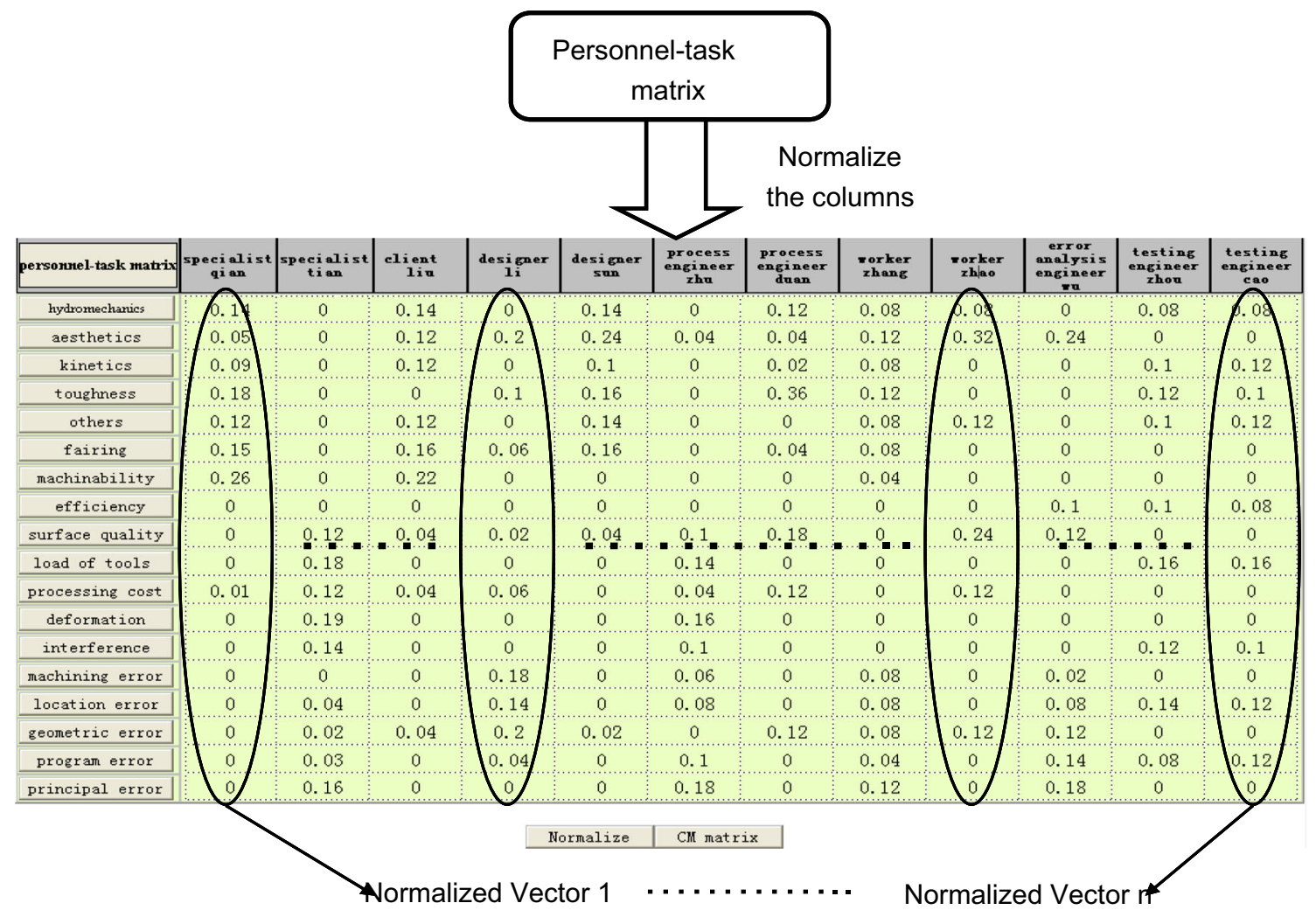

Fig. 6 Personnel-task matrix 


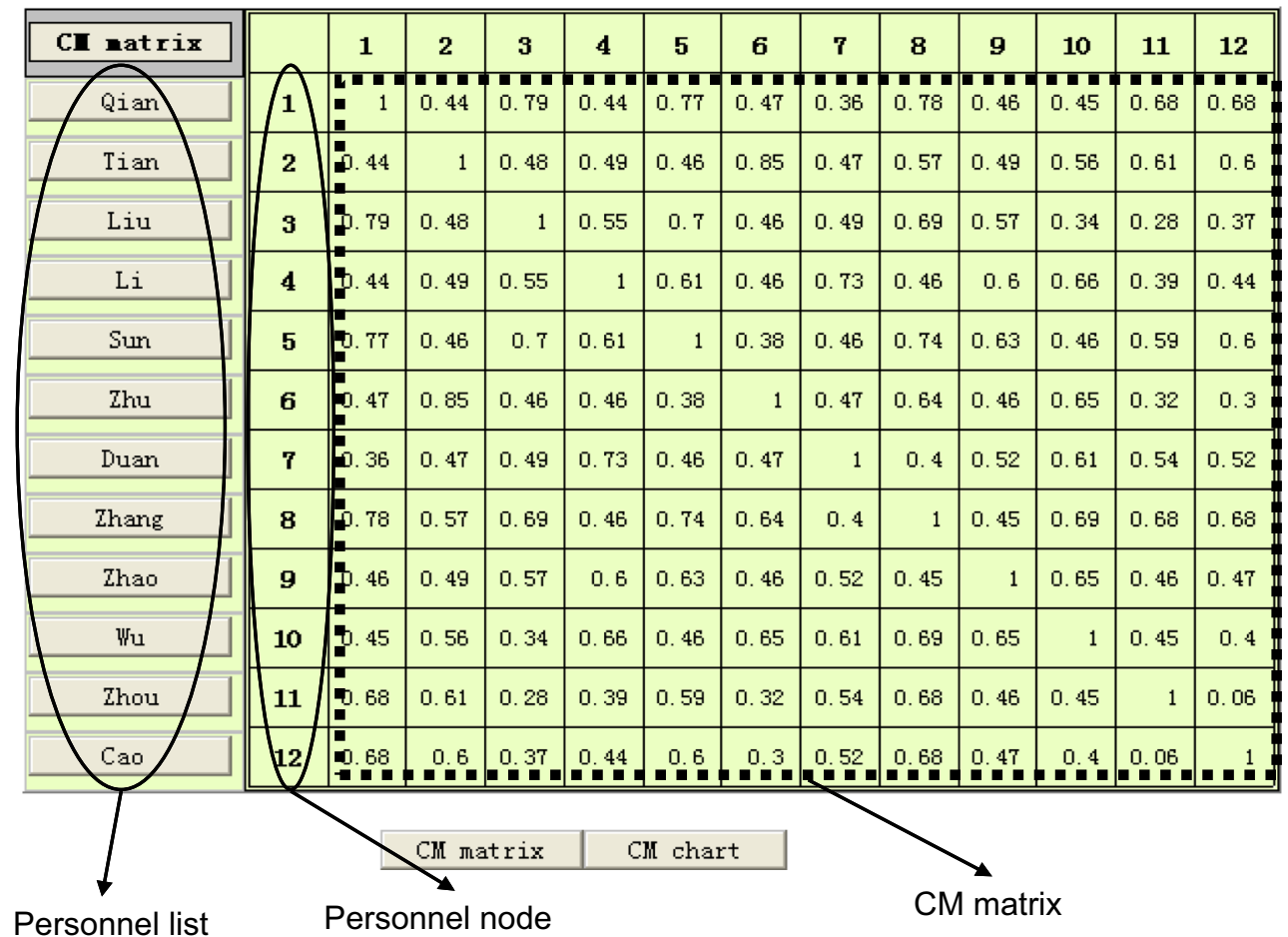

Fig. 7 Correlation matrix of collaborative personnel

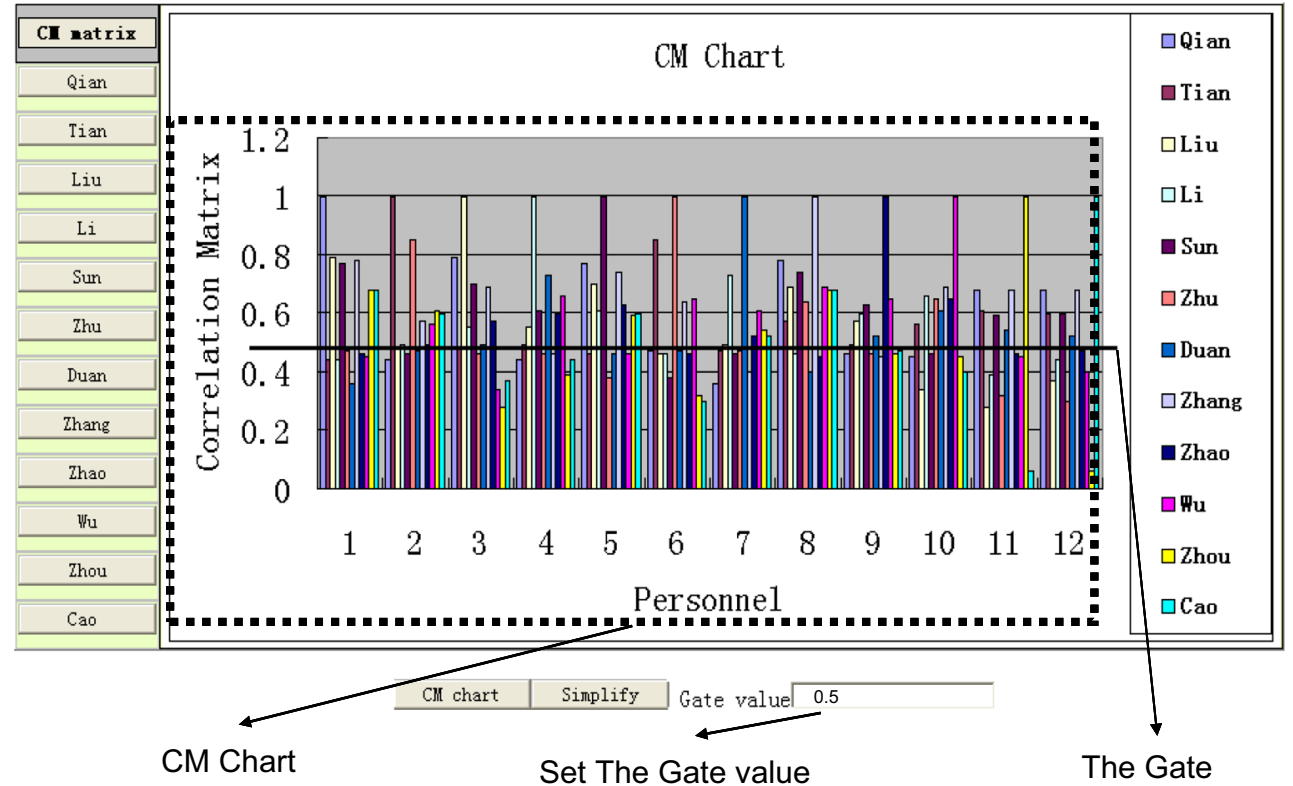

Fig. 8 Simplify the correlation

\section{Correlation matrix}

To simplify the correlations among personnel, a gate is used (shown in Fig. 8). If the correlation intensity is above the gate, the correlations remain and are marked as 1, else, the correlations are ignored and marked as 0 . In this case, the gate value is set as 0.5 . The correlation intensity of specialist Tian and specialist Qian is 0.44 , while the correlation intensity of specialist Qian and client Liu is 0.78 . Using the gate then, the relation between Tian and Qian is marked as 0 , and the one between Qian and Liu is marked as 1 . The gate can be higher or lower by setting the gate value as shown in Fig. 8. After simplification of the correlation matrix, we have a simplified correlation matrix 
whose cells are marked only 0 or 1 (as shown in Fig. 9). Setting the number of sub-spaces as 4 , the system calculates the optimal CSD. In the CSD, there are four sub-

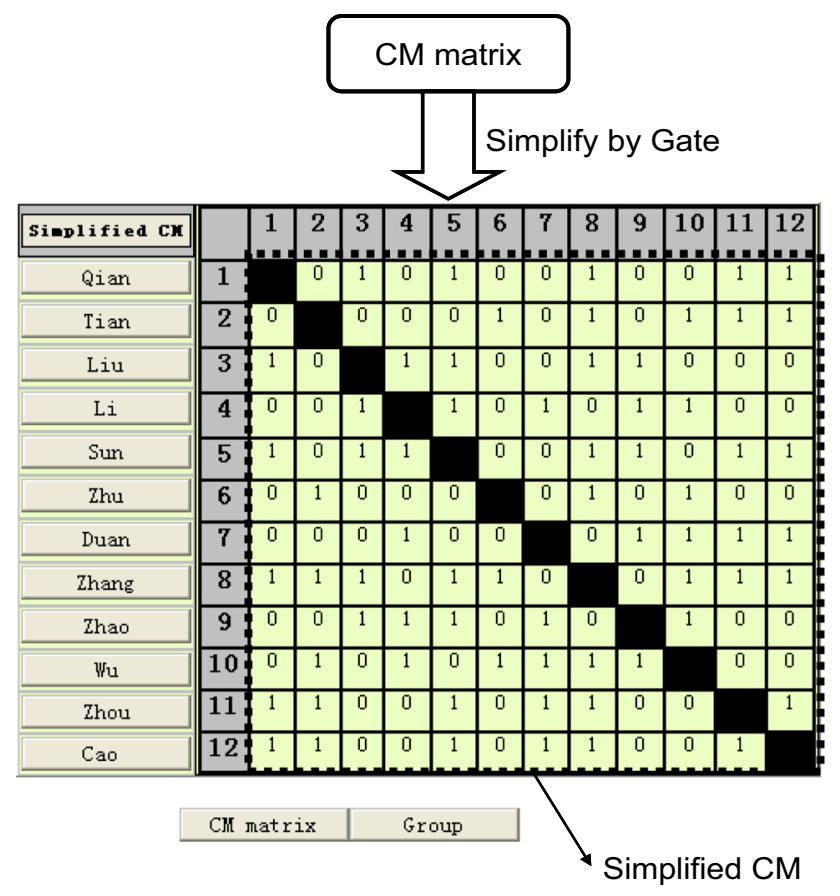

Fig. 9 Simplified correlation matrix of collaborative personnel spaces (shown in Fig. 10), Sub-space 1 (Qian, Sun, Liu and Zhang), Sub-space 2 (Zhang, Tian, Zhu and Wu), Subspace 3 (Wu, Li, Zhao and Duan), Sub-space 4 (Duan, Zhou and Cao). The grey areas depict the crossover of the various sub-spaces. There are no public persons who belong to all of the sub-spaces.

\section{Conclusions}

This paper firstly reviews the literature related to personnel correlation in collaborative product development. We then presented the concept of collaboration space and collaboration space division (CSD). The MDL principle is used to set up an optimal objective function for CSD and then the MDL-based objective function was adapted to a GA, which was used to divide the collaboration space. The GA algorithm encoded the spatial information as a chromosome in binary. After repetitious crossover, mutation, selection and multiplication, a robust chromosome is obtained and subsequently decoded into an optimal collaboration space division. Finally, in the CSD for impeller collaborative development, an $18 \times 12$ personnel-task matrix was set up in depicting the activities of engineers. After calculating the correlation coefficient among personnel, a $12 \times 12$ correlation matrix was obtained to express the relation

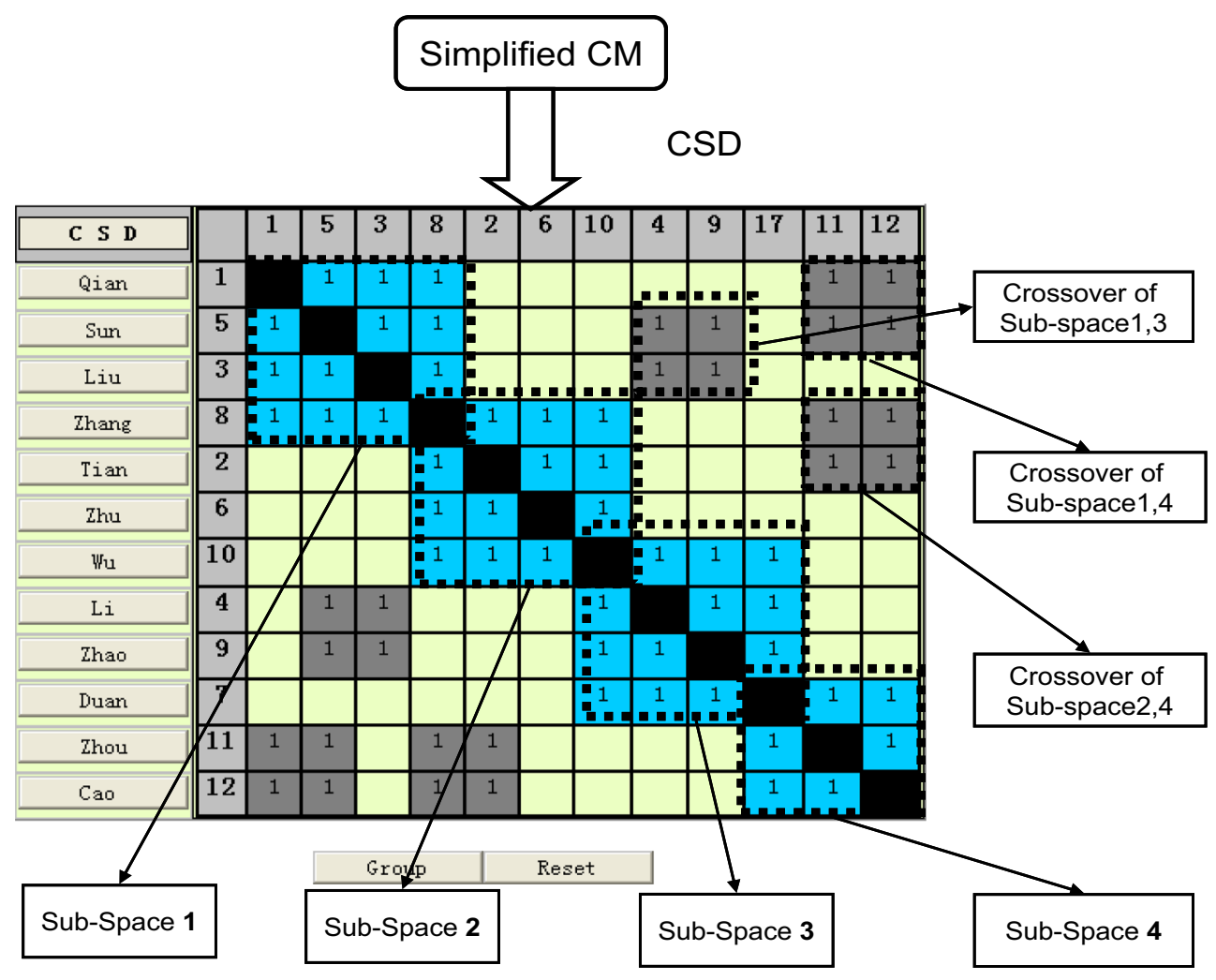

Fig. 10 Result of collaboration space division 
between these engineers. Applying the GA-based CSD, the whole space was divided into four sub-spaces. The new method calculated the members in each sub-space and the sub-spaces of the CS. Furthermore, the intersection of subspaces and public personnel belonging to each sub-space were determined simultaneously.

The methodology presented in this paper is capable of grouping the stuff into teams in an optimal manner and may help to overcome many of the difficulties inherent in organizing personnel. However, the method presented in this paper also simplified the correlation matrix into a zeroone matrix before grouping the personnel into different sub-spaces. The correlation intensity information among members in the same sub-space is lost. Future work will consider the correlation intensity of these members as well.

Open Access This article is distributed under the terms of the Creative Commons Attribution 4.0 International License (http://creative commons.org/licenses/by/4.0/), which permits unrestricted use, distribution, and reproduction in any medium, provided you give appropriate credit to the original author(s) and the source, provide a link to the Creative Commons license, and indicate if changes were made.

\section{References}

Babazadeh R, Razmi J, Ghodsi R (2012) Supply chain network design problem for a new market opportunity in an agile manufacturing system. J Ind Eng Int 8:19

Barrett S, Knosynski B (1982) Inter-organization information sharing system. MIS Q 6(4):93-105

Chiang TA, Trappey AJC, Ku CC (2006) Using a knowledge-based intelligent system to support dynamic design reasoning for a collaborative design community. Int J Adv Manuf Technol 31:421-433

Choi Y, Kim K, Kim C (2005) A design chain collaboration framework using reference models. Int J Adv Manuf Technol 26:183-190

Corbett C, Blackburn J, Wassenhove L (1999) Case study partnerships to improve supply chains. Sloan Manage Rev 40(4):71-82

Cooper RG (1994) Perspective third generation new product process. J Prod Manag 11:3-14

Dao SD, Abhary K, Marian R (2017) An improved genetic algorithm for multidimensional optimization of precedence-constrained production planning and scheduling. Journal of Industrial Engineering International 13(2):143-159

Frecon E, Noeu A (1998) Building distributed virtual environments to support collaborative work. ACM Symposium on Virtual Reality Software and Technology (VRST'98), Taipei

Hong SJ (2004) Web service oriented collaborative workflow management for design and manufacturing. Master Thesis, Michigan State University, East Lansing
Huang CJ, Trappery A, Yao YH (2003) Collaborative design collaborative product commerce, configuration management workflow management. J Chin Inst Ind Eng 20(4):422-434

Kim H, Kim HS, Lee JH et al (2006) A framework for sharing product information across enterprises. Int $\mathbf{J}$ Adv Manuf Technol 27:610-618

Lee DL, Chen YM (1996) Integrated product and process data management. Integr Comput-Aided Eng 13:1-4

Li WD, Qiu ZM (2006) State-of-the-art technologies and methodologies for collaborative product development systems. Int J Prod Res 13:2525-2559

Li WD, Lu YQ, Lu WF et al (2004) Collaborative computer-aided design: research and development status. Comput Aided Des Appl 2:127-136

Ling Ling, Yujin Hu, Wang Xuelin, Li Chenggang (2007) An ontology-based method for knowledge integration in a collaborative design environment. Int $\mathbf{J}$ Adv Manuf Technol 34:843-856

Lu SC, Cai J, Burkett W, Udwadia F (2000) A methodology for collaborative design process and conflict analysis. Ann RP 49(1):69-73

Lutz R. (2002) Recovering high-level structure of software systems using a minimum description length principle, Artificial intelligence and cognitive science, M. O'Neill, RFE. Sutcliffe, C. Ryan, M. Eaton, NJL Griffith (Eds.): Proceedings of the 13th Irish International Conference, AICS 2002, Limerick, Ireland, Sept. 12, 02

May Andrew, Carter Chris (2004) A case study of virtual team working in the European automotive industry. Int $\mathrm{J}$ Ind Ergon 27:171-186

Morris A (2002) The challenge of collaborative commerce. IEE Rev 48:33-37

Navigli R, Velardi P (2003) Ontology learning and its application to automated terminology translation. IEEE Intell Syst 18(1):22-31

Park M, Cutkosky MR (1999) Framework for modeling dependencies in collaborative engineering processes. Res Eng Design $11: 84-102$

Roche C (2000) Corporate ontologies and concurrent engineering. J Mater Process Technol 107:187-193

Summers JD, Butler AC, Kuo E (1999) Development of a feature based design system using virtual reality. In: Proceedings of the 1999 ASME Design Technical Conference and Computers in Engineering Conference. Las Vegas, Nevada, September 11-15

Twigg D (1995) Design chain management. Inter-organisational coordination of product development in the UK automotive industry. Dissertation, University of Warwick

Wang L, Shen W, Xie H, Neelamkavil J, Pardasani A (2002) Collaborative conceptual design-state of the art and future trends. Comput-Aided Des 34(13):981-996

Waterson A, Preece A (1999) Verifying ontological commitment in knowledge-based systems. Knowl-Based Syst 12(1-2):45-54

Wognum PM, Fisscher AM, Weenink AJ (2002) Balanced relationship: management of client-supplier relationships in product development. Technovation 2:34-351

Singhal S, Zyda M (2004) Networked virtual environments. Design and implementation 